\title{
Anaphylatic Shock with Off-Label use of Oxaliplatin in Ovarian Cancer: A Rare Case Report
}

\author{
Abhirami Azad*, Maria Joseph, Minnu Jayamon Biju, Meby Susan Mathew
}

Department of Pharmacy Practice, Nirmala College of Pharmacy, Muvattupuzha, Kerala, INDIA.

\begin{abstract}
Oxaliplatin is a third-generation platinum compound that acts on DNA (Deoxyribonucleic acid) to form inta/inter stand cross-linking and thereby affecting DNA base pairing, replication, gene transcription, and leading to cell death. Anaphylactic reactions associated with platinum compounds are potentially life threatening. A 67-year-old female patient, a known case of carcinoma ovary, previously underwent surgery and multiple lines of chemotherapy now presented with progressive disease. She got admitted for evaluation and management. She recently presented with complaints of fever and fatigue. The patient had a history of allergy to carboplatin. After pre-chemo evaluation, the first cycle of chemotherapy was started with the combination of Cyclophosphamide, Oxaliplatin, and Bevacizumab. Sudden after the administration of Oxaliplatin patient had developed fatigue, weakness along breathing difficulty, change of sound, and cyanosis. The patient was shifted to MICU (Medical Intensive Care Unit) and managed. After that remaining chemotherapy was administered successfully and the patient got discharged. This case highlights the consequence of Oxaliplatin induced anaphylactic shock which is rare but potentially fatal and so oncologists should be vigilant when patients have signs or symptoms similar to anaphylactic reactions.
\end{abstract}

Key words: Oxaliplatin, Ovarian cancer, Chemotherapy, Platinum compounds, Anaphylactic reactions.

\section{INTRODUCTION}

Oxaliplatin is a third-generation platinum compound approved by the FDA for the treatment of colorectal cancer. Since phase II studies in metastatic breast cancer have shown some efficacy with oxaliplatin. ${ }^{1,2}$ This medication is one of the rare broad-spectrum agents that act with DNA to form inta/inter stand crosslinking and affecting DNA base pairing, replication, gene transcription leading to cell death. ${ }^{3}$ This platinum complex differs significantly from cisplatin by targeting different biomolecules. Oxaliplatin does not easily develop resistance and remains active against cancerous tissue even in the presence of resistance to cisplatin. Anaphylactic reactions related to platinum compounds are potentially life-threatening.
These undesirable reactions produced by the immune system are most serious and can lead to death without adequate medical attention. Anaphylactic reactions were found to be less common with oxaliplatin when compared with other platinum compounds. Currently, Oxaliplatin is used off-label in various malignancies leading to an increased incidence of hypersensitivity reactions $(10-12 \%$, ) with the rate of severe reactions $>1 \%{ }^{4}$

\section{CASE DESCRIPTION}

A 67-year-old female patient, a known case of carcinoma of the ovary, previously underwent surgery and received multiple lines of chemotherapy as she was presented with progressive disease on evaluation.
DOI: 10.5530/ijopp.14.4.66

Address for correspondence: Ms. Abhirami Azad, Pharm.D, Intern, Nirmala College of Pharmacy, Muvattupuzha-686661, Kerala, INDIA.

Phone no: +91 8301020179 Email id: abhiramiazad111@ gmail.com

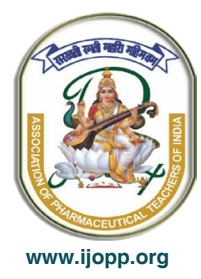


The patient had a history of delayed allergic reaction to carboplatin which she develops during the third cycle of treatment two years ago. She experiences symptoms like rash and abdominal pain. After that treatment was replaced with Cisplatin and was successfully completed. She had a recurrence after 7 months for which she was treated with Lipodox (Doxorubicin). Again with the progression of the disease after 6 months she was treated with Nab-Paclitaxel and Bevacizumab and then maintained on Fluorouracil. She recently presented with complaints of fever and fatigue. However, her temperature was found to be elevated and other vitals were found to be normal. She was treated with oral antibiotics-Tab. Levoflox, Tab Pantoprazole, Tab. Paracetamol and IV Normal saline. Computed tomography (CT) on Abdomen and pelvis showed mild to moderate partly loculated ascites with multiple peritoneal deposits. She was planned for chemotherapy with a combination of Cyclophosphamide, Oxaliplatin, and Bevacizumab. Cardiology consultation was given for fitness. An echocardiogram (ECHO) was taken and it showed no regional wall motion abnormalities (RWMA), good left ventricle (LV) systolic function. After obtaining the consent and pre-chemo evaluation first cycle of chemotherapy was started with the combination of Cyclophosphamide, Oxaliplatin, and Bevacizumab. Soon after the administration of Oxaliplatin patient developed fatigue, weakness, breathing difficulty with a respiratory rate of 28 breaths/min, change of sound, and cyanosis. On laboratory investigation her $\mathrm{HB}$ (hemoglobin) count as well serum potassium level was found to be low whereas WBC (White Blood Cell) total count was found to be elevated. The patient was diagnosed with anaphylactic shock induced by Oxaliplatin. The patient was shifted to MICU (Medical Intensive Care Unit) and managed with Inj. Adrenaline $1 / 1000(1 \mathrm{ml})$, Inj. calcium gluconate $(1 \mathrm{amp}$ in $100 \mathrm{ml} \mathrm{NS}$ over $30 \mathrm{~min}$ ), Inj. magnesium sulfate (1 amp in $100 \mathrm{ml} \mathrm{NS}$ over $60 \mathrm{~min}$ ) and salbutamol nebulizer. The patient's condition improved and was shifted to the ward. After that remaining chemotherapy was administered successfully and the patient got discharged. At the time of discharge, the patient was better and hemodynamically stable.

\section{DISCUSSION}

Several studies have shown that the estimated incidence of Oxaliplatin-induced serious adverse reactions is less than $2 \% .{ }^{2,5}$ The study conducted by J-L Misset, ${ }^{6}$ pointed out the safety profile of Oxaliplatin was better than cisplatin when considering gastrointestinal, neurosensory hematological, and renal side effects in patients treated for ovarian cancer. The precise mechanism of Oxaliplatin hypersensitivity is not clearly understood, although it is considered to be linked with type 1 immunoglobulin E-mediated reaction that causing the release of chemical mediators, leading to vasodilation and edema. In vitro and in vivo studies depicted the lack of cross-resistance between cisplatin and carboplatin in the human ovarian cell line. ${ }^{7}$ However, in this study, the patient had a history of allergy to carboplatin. Parel M et al. ${ }^{8}$ in their study showed that anaphylaxis was more frequent in women although the reason for increased risk was unknown. They also mentioned that all females manifested acute hypokalemia which was similar to this study. Another study conducted by Thomas R.R et al., pointed out that the same symptoms have recurred after rechallenge and that leads to the discontinuation of the drug. Unlike our study where rechallenge was not performed after recovery. Skin tests to exclude crossreactivity before substituting one platinum analog to another was suggested by Mayer et al., ${ }^{10}$ in their study which was not performed in this study.

\section{CONCLUSION}

The rare and fatal complication due to anaphylactic shock induced by Oxaliplatin can lead to death in the absence of prompt medical attention. Although Oxaliplatin is sporadically associated with this reaction, very few patients tolerate this complication irrespective of desensitization thereby necessitating the change in drug regimen. It should also be noted that rechallenge with this medication would be a challenge. Moreover, the mechanism underlying Oxaliplatin induces serious adverse reactions is unclear. Hence clinicians need to be cautious prescribing this medication for the overall well-being of patients.

\section{ACKNOWLEDGEMENT}

We are thankful to the Management, Pharmacy Practice Department, and the Principal of our college for immense support and encouragement.

\section{CONFLICT OF INTEREST}

The authors declare that they have no competing interests.

\section{ABBREVIATIONS}

DNA: Deoxyribonucleic Acid; MICU: Medical Intensive Care Unit; CT: Computed tomography; ECHO: 
Echocardiogram; RWMA: Regional Wall Motion Abnormalities; LV: Left Ventricle; HB: Hemoglobin; WBC: White Blood Cell.

\section{REFERENCES}

1. Delpeuch A, Leveque D, Rob L, Bergerat JP. Off-label use of oxaliplatin in patients with metastaticbreastcancer. Anticancer Res. 2011;31(5):1765-7. PMID 21617237.

2. Culy $C R$, Clemett $D$, Wiseman LR. Oxaliplatin. A review of its pharmacological properties and clinical efficacy in metastatic colorectal cancer and its potential in other malignancies. Drugs. 2000;60(4):895-924. doi: 10.2165/00003495200060040-00005, PMID 11085200.

3. Wang JH, King TM, Chang MC, Hsu CW. Oxaliplatin-induced severe anaphylactic reactions in metastatic colorectal cancer: case series analysis. World J Gastroenterol. 2012;18(38):5427-33. doi: 10.3748/wjg.v18. i38.5427, PMID 23082060.

4. Lenz HJ. Management and preparedness for infusion and hypersensitivity reactions. Oncologist. 2007;12(5):601-9. doi: 10.1634/theoncologist.12-5-601, PMID 17522249.
5. Lee MY, Yang MH, Liu JH, Yen CC, Lin PC, Teng HW, Wang WS, Chiou TJ, Chen PM. Severe anaphylactic reactions in patients receiving oxaliplatin therapy: a rare but potentially fatal complication. Support Care Cancer. 2007;15(1):89-93. doi: 10.1007/s00520-006-0107-9, PMID 16865410.

6. Misset JL. Oxaliplatin in practice. BrJ. Br J Cancer. 1998;77;Suppl 4:4-7. doi: 10.1038/bjc.1998.428, PMID 9647612.

7. Pendyala L, Creaven PJ. In vitro cytotoxicity, protein binding, red blood cell partitioning, and biotransformation of oxaliplatin. Cancer Res. 1993;53(24):5970-6. PMID 8261411.

8. Parel M, Ranchon F, Nosbaum A, You B, Vantard N, Schwiertz V, et al. Hypersensitivity to oxaliplatin: clinical features and risk factors. BMC Pharmacol Toxicol January 132014. 2014;15:1. doi: 10.1186/2050-6511-15-1, PMID 24417770.

9. Thomas RR, Quinn MG, Schuler B, Grem JL. Hypersensitivity and idiosyncratic reactions to oxaliplatin. Cancer. 2003;97(9):2301-7. doi: 10.1002/cncr.11379, PMID 12712487

10. Meyer L, Zuberbier T, Worm M, Oettle H, Riess H. Hypersensitivity reactions to oxaliplatin: cross-reactivity to carboplatin and the introduction of a desensitization schedule. J Clin Oncol. 2002;20(4):1146-7. doi: 10.1200/ JCO.2002.20.4.1146, PMID 11844841. 